\title{
Diffusion coefficient of Brownian particle in rough micro-channel
}

\author{
Chen Jun ${ }^{a}$, X.F. Peng ${ }^{\mathrm{a}, *}$, D.J. Lee $^{\mathrm{b}}$ \\ a Thermal Engineering Department, Tsinghua University, Beijing 100084, China \\ $\mathrm{b}$ Department of Chemical Engineering, National Taiwan University, Taipei 10617, Taiwan \\ Received 16 July 2005; accepted 10 September 2005 \\ Available online 28 November 2005
}

\begin{abstract}
This study examines the feasibility of using of the lattice Boltzmann method to determine how the surface roughness of a quadrate channel affects the diffusion coefficient of Brownian particle(s). The surface was represented by a regular array of spheres. Surface roughness reduced the diffusion coefficient of the Brownian particle(s) because of a change in the velocity autocorrelation function decay and in pressure. Additionally, the neighboring particles increased the diffusion coefficient of Brownian particle.
\end{abstract}

(c) 2005 Elsevier Inc. All rights reserved.

Keywords: Diffusion coefficient; Surface roughness; Lattice Boltzmann method; Velocity autocorrelation function; Quadrate channel

\section{Introduction}

Diffusion of particles in a micro-channel, also called hindered diffusion or restricted diffusion, occurs in many applications, including capillary electrophoresis and diffusion in porous media. Micro-scale confinement reduces diffusion coefficient of a particle [1-3]. Interaction between a solid wall and suspended particles had been studied using dynamic-light scattering [4], video microscopy [5] and digital imaging [6]. Benesch and Yiacoumi [7] theoretically evaluated the perpendicular and parallel diffusion coefficients of a single particle in a confined environment using the method of reflection. The prediction of their model was consistent with experimental data.

The motion of a colloidal particle between confined walls was simulated using the lattice Boltzmann method (LBM), which showed the presence of a negative algebraic tail of the velocity correlation function decay [8,9], caused by the diffusive decay of density perturbations. Stepisnik and Callaghan [10] later experimentally verified using modulated gradient spinecho NMR.

No wall is perfectly smooth. The effects of surface roughness on the motion of a particle may become significant when the characteristic scale of the confinement is small. To our

\footnotetext{
* Corresponding author.

E-mail address: djlee@ntu.edu.tw (X.F. Peng).
}

knowledge, no work has examined how the surface roughness of a micro-channel affects the diffusion coefficient of single or many Brownian particles. This study explores how surface roughness affects the diffusion coefficients of Brownian particle(s). The Navier-Stokes equation of fluid between particles and confinement walls is determined using LBM [11].

\section{The lattice Boltzmann method}

In contrast to conventional numerical schemes, which discretize the macroscopic continuum equation to finite difference forms, the lattice Boltzmann method is based on microscopic model and mesoscopic kinetic equations.

The Boltzmann equation with single relaxation time approximation is

$\frac{\partial f}{\partial t}+\zeta \cdot \nabla f=-\frac{1}{\lambda}\left[f-f^{(\mathrm{eq})}\right]$,

where $\zeta$ is the particle velocity; $f^{(\mathrm{eq})}$ is the equilibrium distribution function, and $\lambda$ is the relaxation time. Discretizing Eq. (1) in the velocity space $\zeta$ using a finite set of velocities $\mathbf{e}_{i}$, yields

$\frac{\partial f_{i}}{\partial t}+\mathbf{e}_{i} \cdot \nabla f_{i}=-\frac{1}{\lambda}\left[f-f_{i}^{(\mathrm{eq})}\right]$.

For a square lattice of two dimensions, $\mathbf{e}_{0}=(0,0), \mathbf{e}_{i} .=$ $c[\cos \pi(i-1) / 2, \sin \pi(i-1) / 2]$ with $i=1-4$, and $\mathbf{e}_{i}=$ $c[\cos \pi(2 i-1) / 4, \sin \pi(2 i-1) / 4]$ for $i=5-8$, are the nine 
possible velocity vectors (D2Q9), and the equilibrium distribution functions are of the form,

$f_{i}^{(\mathrm{eq})}=\alpha_{i} \rho\left[1+\frac{3}{c^{2}} \mathbf{e}_{i} \cdot \mathbf{u}+\frac{9}{2 c^{4}}\left(\mathbf{e}_{i} \cdot \mathbf{u}\right)^{2}-\frac{3}{2 c^{2}} u^{2}\right]$.

In Eq. (3), $\alpha_{0}=4 / 9, \alpha_{1}=\alpha_{2}=\alpha_{3}=\alpha_{4}=1 / 9$ and $\alpha_{5}=$ $\alpha_{6}=\alpha_{1}=\alpha_{s}=1 / 36 ; c=\delta x / \delta t$ is the lattice speed, and $\delta x$ and $\delta t$ are the lattice constant and the time step, respectively. The density $\rho$ and the velocity $\mathbf{u}$ are given by

$\rho=\sum_{i} f_{i}, \quad \mathbf{u}=\sum_{i} f_{i} \mathbf{e}_{i} / \rho$.

The lattice Boltzmann equation is obtained by further discretizing Eq. (2) in space and time as follows:

$f_{i}\left(\mathbf{x}+\delta x \mathbf{e}_{i}, t+\delta t\right)-f_{i}(\mathbf{x}, t)=\frac{1}{\tau}\left(f_{i}-f_{i}^{(\mathrm{eq})}\right)$,

where $\tau=\lambda / \delta t$. The macroscopic equations and the NavierStokes equation, are given by the Chapman-Enskog procedure. The viscosity in the macroscopic equation is

$\nu=\frac{2 \tau-1}{6} c^{2} \delta t$.

Herein, $\delta x=\delta t=c=1$ is set. In the simulation of solid particles, Ladd's model $[12,13]$ is used to specify the nonslip boundary conditions at the solid-liquid boundaries and the suitable hydrodynamic force on the solid.

\section{System of interest}

The channel is filled with quiescent Newtonian fluid. The height and the width of the channel are both $h$. A Brownian particle is placed at the center of the channel. At $t=0$, the particle moves at a velocity of $v(0)$. The time evolution of a particle's velocity $v(t)$ is determined according to an LBM scheme. Linear response theory dictates that the relaxation function, $v(t) / v(0)$, is identical to the normalized velocity autocorrelation function (VACF) for the Brownian motion of a particle in thermal equilibrium [14].

Figs. 1 and 2 display channels with smooth and rough surfaces, respectively. A Brownian particle with nonslip boundaries was located at the center of the channel. The surfaces of the channel walls were considered to be nonslip. The two far ends of channel ( $x$-axis) were periodic boundaries. In the simulation of a single particle, the channel length $(l)$ was 1000 times $h$ to ensure that the periodical boundary conditions did not influence the simulation results. When the length of the channel was finite, however, the interactions among neighboring spheres separated by a distance $l$, and moving together, could be determined.

The lattice spacing and the time step were both set to unity. The kinematic viscosity $v$ of the fluid was $1 / 6$ and density $\rho$ was 36 . The Brownian particle had a diameter $(a)$ of 9 and was neutrally buoyant. An array of spheres of radii 2.5 was located at the surface of the wall to simulate its roughness. Initially, the velocity of particle $v(0)$ was set to 0.001 , similar to the rootmean-square velocity of the Brownian motion. The subsequent motion of the particle and the fluid was numerically determined

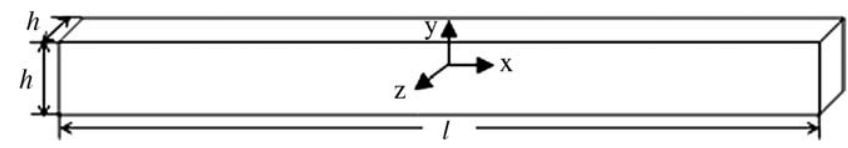

Fig. 1. The smooth channel under investigation.


Fig. 2. The rough channel under investigation.

for 1000 time steps, during which the effect of the particle's motion had not propagated to the far ends with periodic boundary conditions.

Based on the evolution of the particle velocity, the diffusion coefficient $(D)$ was evaluated using the Green-Kubo integral (Eq. (7)):

$D=\frac{1}{3} \int_{0}^{\infty} \mathrm{d} t\langle v(0) v(t)\rangle$.

The term $\langle v(0) v(t)\rangle$ is the VACF determined from the time evolution of particle velocity. The parallel diffusion coefficient $D_{\|}$ was determined from the velocity along the $x$-axis. The perpendicular diffusion coefficient $D_{\perp}$ could be obtained when the velocity was normal to the wall along the $y$-axis. Note that the units adopted are omitted herein but are scalable to different systems if only the particles considered are Brownian and the surrounding fluid can be taken as Newtonian. For instance, if the units of length and time are $\mu \mathrm{m}$ and $\mathrm{s}$, respectively, then the diffusivity obtained is in $\mu \mathrm{m}^{2} / \mathrm{s}$.

\section{Results and discussion}

\subsection{Diffusion coefficient}

Fig. 3 presents the calculated parallel and perpendicular diffusion coefficients in smooth and rough channels, $a / h$ is the ratio of the particle diameter to the channel height, and $D / D_{0}$ is the ratio of the diffusion coefficient in the channel to the Stokes-Einstein value (Eq. (8)) of an unbound fluid. In this work, $D_{0}$ is determined to be $2.7 \times 10^{5}$.

$D_{0}=\frac{k_{B} T}{3 \pi \rho v a}=\frac{m v^{2}(0)}{3 \pi \rho v a}=\frac{a^{2} v^{2}(0)}{18 v}$.

The perpendicular diffusion coefficients all exceeded their parallel counterparts.

Also, the surface roughness reduced the diffusion coefficients, to a greater extent as $a / h$ fell. Restated, when the par- 


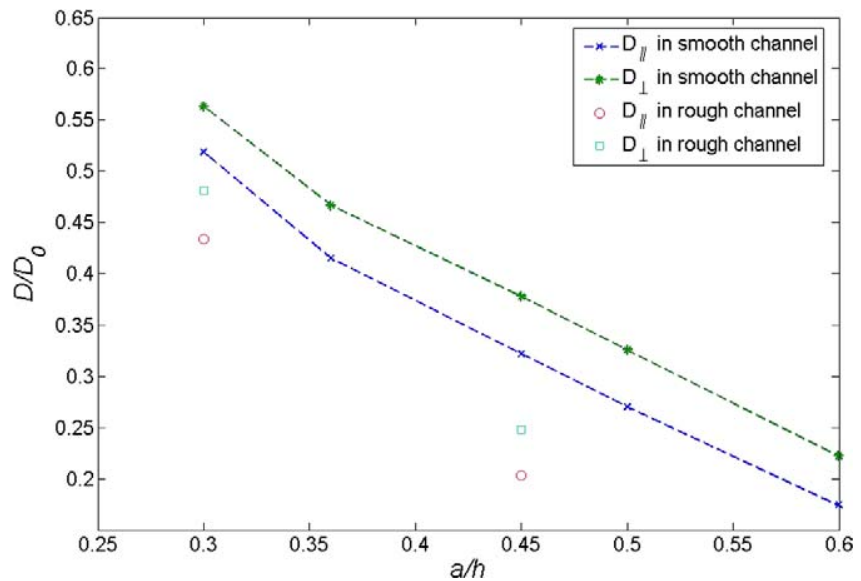

Fig. 3. Diffusion coefficients in rough and smooth micro-channel. (a)



(b)

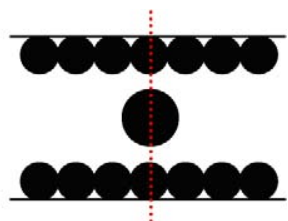

Fig. 4. The position of Brownian particle relative to surface spheres: (a) staggered, (b) in-line.

ticle size approached the channel size, the rigid, rough walls dominated the restriction of the Brownian diffusion.

The $D_{\|}$values were calculated for smooth channels of dimensions $1000 \times 25 \times 25$ and $10000 \times 25 \times 25$, to be $8.7 \times 10^{-6}$, and $8.4 \times 10^{-6}$, respectively, to verify the infinite channel approximation. Other simulations yielded a similar, relative error with channels of various lengths. Therefore, the possible errors associated with the finite length of the channel were under $4 \%$.

Whether the initial position of the Brownian particle initially affected the diffusion coefficients was also studied. Simulations with the particle's center "staggered" from (Fig. 4a) or "in-line" with (Fig. 4b) the centers of the spheres showed that the initial position of the particle did not noticeably affect the diffusion coefficients. Such a conclusion was somewhat surprising because the surface roughness did affect the diffusion coefficient (Fig. 3).

\subsection{Normalized velocity autocorrelation function (VACF)}

The diffusion coefficient was determined from VACF using the Green-Kubo integral (7). Therefore, the effects of wall roughness could be determined from the change in VACF when the surface of the wall was rough.

Figs. 5 and 6 compare the VACF decays in a rough channel and a smooth channel, for parallel and perpendicular diffusions, respectively.

As the particle was moving parallel to the channel wall, the VACF fell to a minimal, negative VACF, after short time before slowly approaching zero at the long-time limit (Fig. 5). This negative long-time tail of VACF has been reported elsewhere [8-10]. Reducing the height of the channel causes the

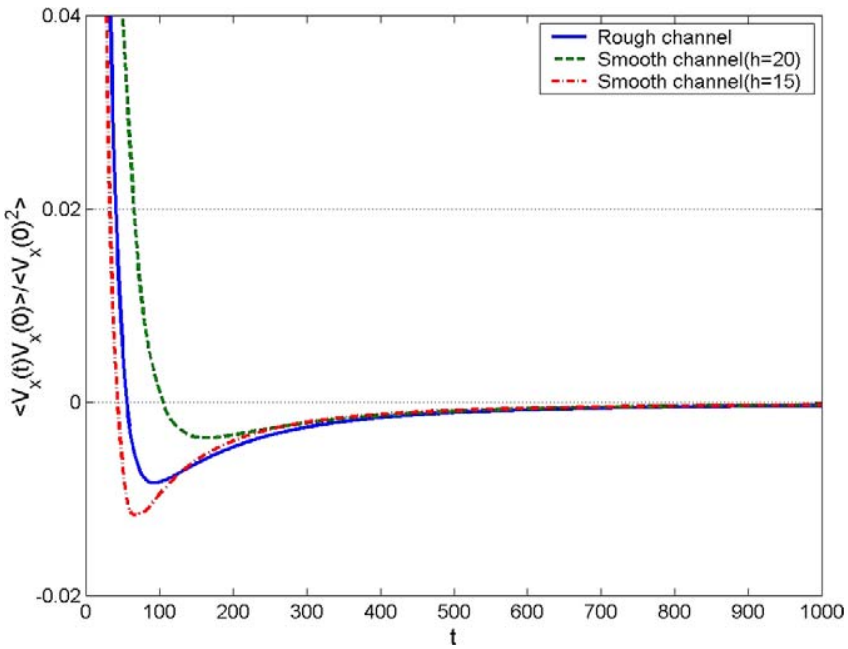

Fig. 5. The VACF of particle motion in parallel with channel wall.

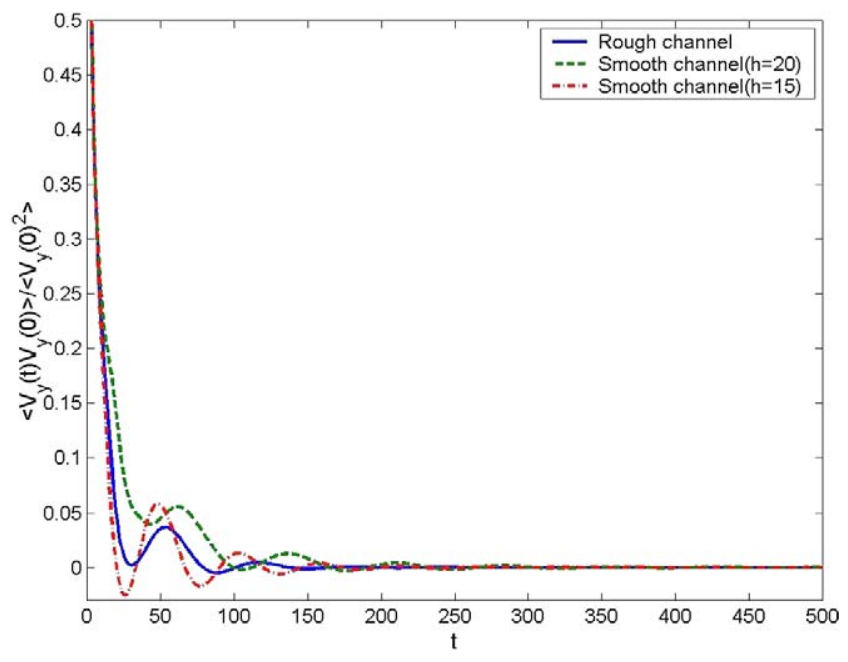

Fig. 6. The VACF of particle motion perpendicular to channel wall.

VACF to drop faster, reaching more negative autocorrelation value. Meanwhile, the rough surface with $h=20$ located between tests with $h=15$ and 20. Restated, the rough surface can be approximated by a smooth channel with a lower height. Close observation showed that the negative segment of VACF decayed more slowly in the rough channel, which decay was responsible for the lower drop in the parallel diffusion coefficient in the rough channel.

When the particle moved perpendicularly to the channel wall, the VACF also fell to a minimum quickly (Fig. 8). However, unlike under parallel flow conditions, the VACF associated with perpendicular flow then oscillated and decayed before reaching zero in the long-time limit. The main difference between the rough and smooth channels was the intensity of oscillation of VACF. When the particle moved through a rough channel, its amplitude of oscillation was small. Only two obvious oscillations in VACF were observed, while three were observed in the smooth channel case. The weaker oscillation was responsible for the lower perpendicular diffusion coefficient in the rough channel. 



(a)
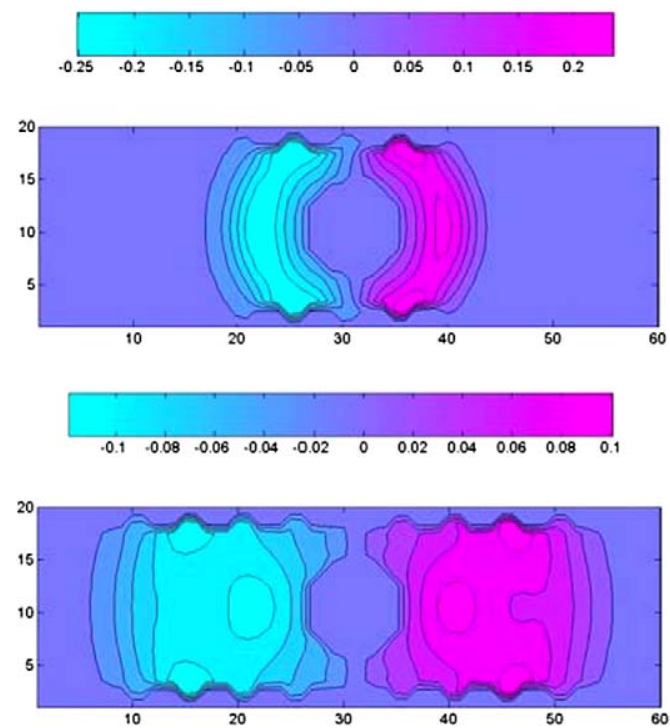
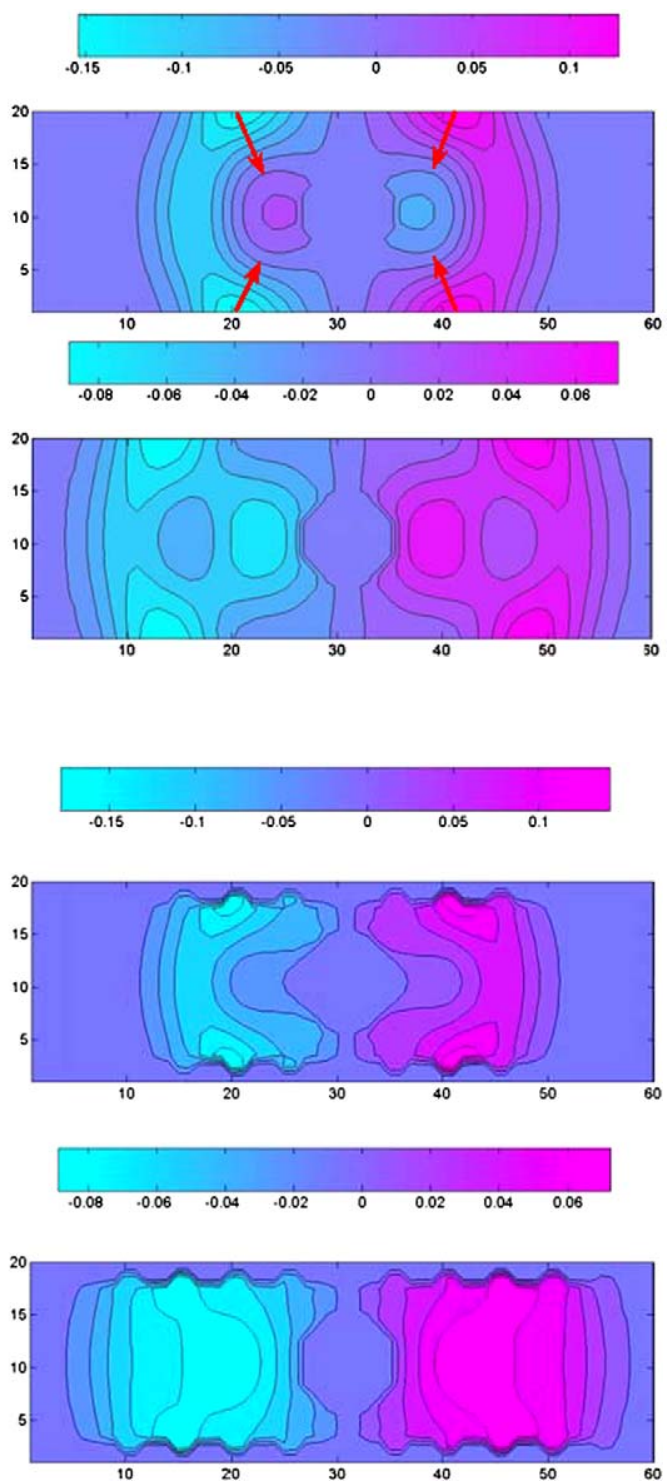

(b)

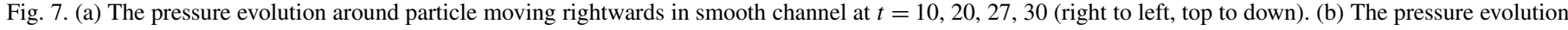
around particle moving rightwards in rough channel at $t=10,20,27,30$ (right to left, top to down).

As the particle moved along the channel to the right, the disturbed liquid propagated outwards, establishing pressure "waves" at higher magnitude ahead of the particle, and at lower magnitude behind it. These pressure waves hit the smooth walls, reflected back, and interfered with other waves to influence the force field around the particle (Fig. 7a). When the walls were rough, more reflected waves were observed, and the interference pattern that interacted with the particle was more complex (Fig. 7b). This nonlinear interaction explained the difference between the VACF curves in Figs. 5 and 6. The time evolutions of pressure waves associated with the motion of a particle toward smooth and rough surfaces were similar, and were plotted in Figs. 8a and 8b. In the perpendicular case, pressure waves yielded propagated along the channel and were reflected. Interactions accelerated the decay of the pressure waves.

\subsection{Diffusion of identical particles}

When infinitely many identical Brownian particles were placed in a micro-channel at fixed separation, they interacted with each other. Changing the length $l$, but keeping all other dimensions unchanged, revealed the effects of particle-particle interactions.

The VACF of a moving particle along the channel wall begins to be affected by the neighboring particles at $t=83,47$ and 14 at $l=60,40$ and 20, respectively (Fig. 9). The presence of neighboring particles always increased the VACF. The adjacent particles tended to move coherently. This phenomenon has also been observed by digital videomicroscopy [15]. Fig. 10 presents the evolution of pressure around a particle. When many identical Brownian particles move together along a channel, all of the particles produce pressure waves and interact with each other. 

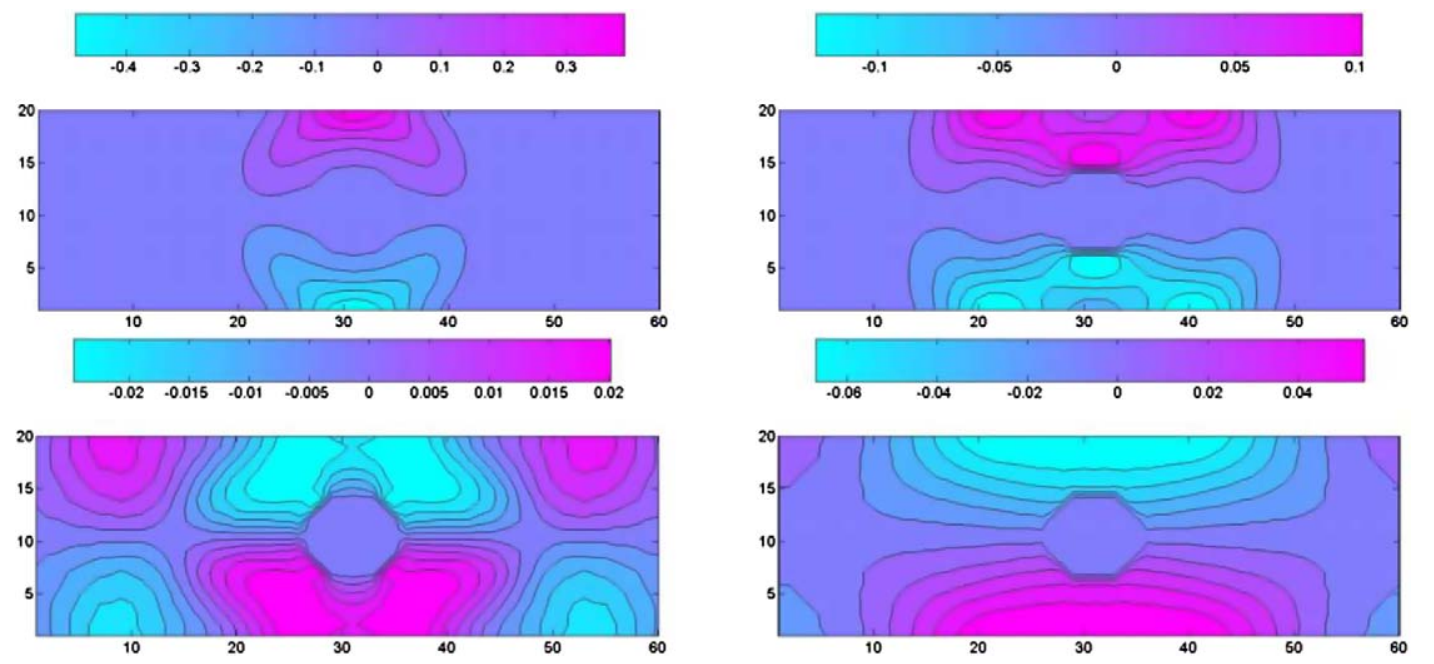

(a)


(b)

Fig. 8. (a) The pressure evolution around particle moving upwards in smooth channel at $t=10,20,40,50$ (right to left, top to down). (b) The pressure evolution around particle moving upwards in rough channel at $t=10,20,30,40$ (right to left, top to down).

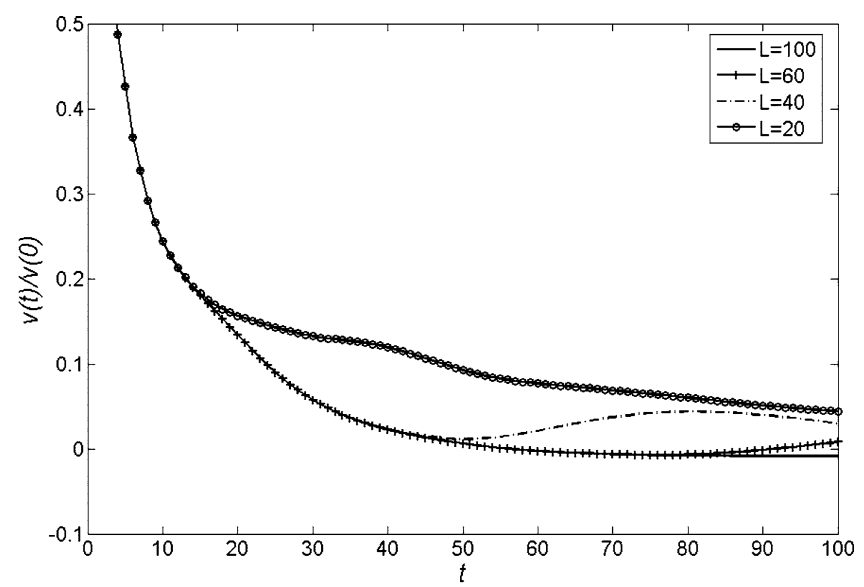

Fig. 9. The VACF of particle motion in channels with different length.
Table 1

Diffusion coefficients of identical particles with separation of $l$ in rough channels, $a / h=9 / 25$

\begin{tabular}{lllllll}
\hline$l$ & 20 & 40 & 60 & 80 & 100 & 1000 \\
\hline$D_{\|}\left(\times 10^{5}\right)$ & 1.61 & 1.06 & 0.87 & 0.77 & 0.72 & 0.55 \\
$D_{\perp}\left(\times 10^{5}\right)$ & 0.64 & 0.67 & 0.67 & 0.67 & 0.67 & 0.67 \\
\hline
\end{tabular}

Both the parallel and perpendicular diffusion coefficients of identical Brownian particles in a micro-channel were evaluated using the same method as was applied to a single particle system (Table 1). The parallel diffusion coefficient increases as the separation among particles decreases, yielding the VACF results. When the channel length was reduced to 20, the parallel diffusion coefficient was almost three times that in the infinitely long channel. However, the perpendicular diffusion coefficient did not depend as strongly on the channel length $l$. Only when 

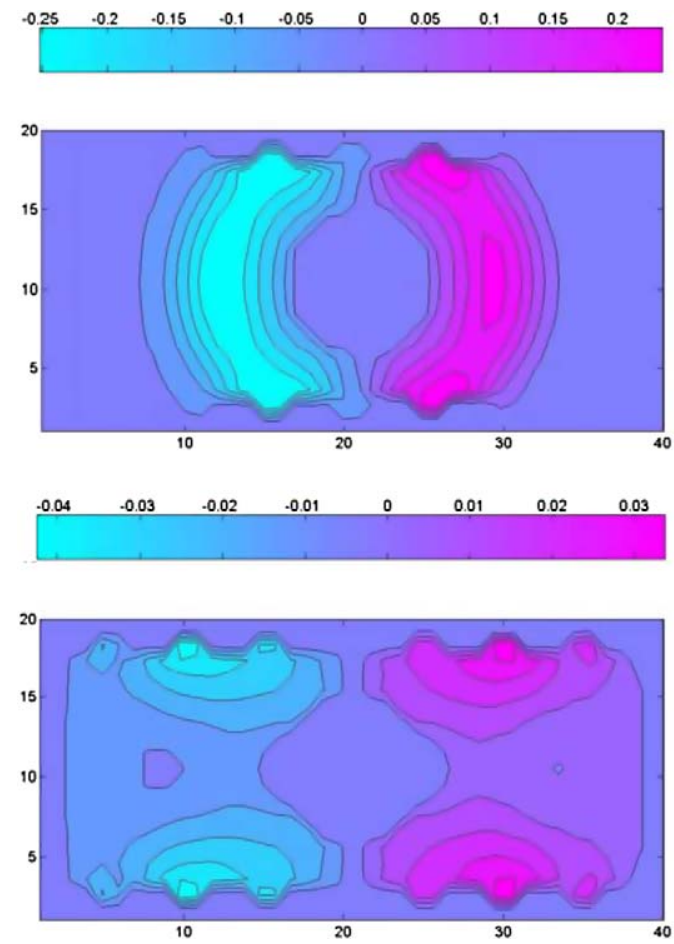


Fig. 10. The pressure evolution around particle in rough channel at $t=10,30,40,60$.

the channel length was below 20 herein did neighboring particles influence the neighboring particles. This occurrence was attributable to the strong dissipation of pressure waves in a zigzag fashion through the channel.

\section{References}

[1] E.L. Cussler, Diffusion, Mass Transfer in Fluid Systems, Cambridge Univ. Press, Cambridge, 1997.

[2] E.M. Renkin, J. Gen. Physiol. 38 (1954) 225.

[3] P.M. Bungay, H. Brenner, Int. J. Multiphase Flow 1 (1997) 25

[4] L. Lobry, N. Ostrowsky, Phys. Rev. B 53 (1996) 12050.
[5] B. Lin, J. Yu, S.A. Rice, Phys. Rev. E 62 (2000) 3909.

[6] L.P. Faucheux, A.J. Libchaber, Phys. Rev. E 49 (1994) 5158.

[7] T. Benesch, S. Yiacoumi, Phys. Rev. B 68 (2003) 21401.

[8] M.H.J. Hagen, I. Pagonabarraga, C.P. Lowe, D. Frenkel, Phys. Rev. Lett. 78 (1997) 3785.

[9] I. Pagonabarraga, M.H.J. Hagen, C.P. Lowe, D. Frenkel, Phys. Rev. E 59 (1999) 4458.

[10] J. Stepisnik, P.T. Callaghan, Phys. B 292 (2000) 296.

[11] S. Chen, G.D. Doolen, Ann. Rev. Fluid Mech. 30 (1998) 329.

[12] A.J.C. Ladd, R. Verberg, J. Stat. Phys. 104 (2001) 1191.

[13] A.J.C. Ladd, J. Fluid Mech. 271 (1994) 285.

[14] J. Horbach, D. Frenkel, Phys. Rev. E 64 (2001) 61507.

[15] B. Cui, H. Diamant, B.H. Lin, Phys. Rev. Lett. 89 (2002) 188302. 